\title{
The Presentation of Gastric Cancer in the Kashmiri Native An Analysis of 850 Cases
}

\author{
Hamid A. Durrani, M.D., F.A.C.G, G.M. Malik, M.D., N.M. Tikku, M.D., Mehraj Ud Din Khan, M.D., \\ Inder Gupta, M.D., Masarrat Durrani, M.B.B.S., \\ Srinagar, Kashmir, India
}

DOI: http://dx.doi.org/10.5915/20-2-13266

\begin{abstract}
Gastric cancer is the most frequent gastrointestinal neoplasm in the native population of Kashmir valley situated in the mountains of North-West India. The prevelence rate in other population groups in India is much lower.

The study is a comprehensive analysis of 850 cases diagnosed in the last five years at one teaching-cum-referral center. Of these, $82 \%$ were males and $18 \%$ were females; $62 \%$ were smokers and $38 \%$ were non-smokers; $60 \%$ of patients belonged to the age group 41-60 years.

Endoscoric appearances were classified as: nodular mass $47 \%$, polypoid mass $20 \%$, malignant ulcer $10 \%$, infilterative lesion $8 \%$, rounded tumor mass $8 \%$, linitis plastica $5 \%$ and 'early carcinoma' $2 \%$. On histopathological typing; $79 \%$ of the lesions were adenocarcinomes, $8 \%$ mucoid carcinomas, $8 \%$ leiomyosarcomes, $4 \%$ schirrous carcinomas, and $1 \%$ reticulum cell sarcomas.

The possible etiological factors peculiar to the dietary constituents, including the consumption of hot salted tea and of a particular leafy vegetable, are singled out. The genetic factors may have an important role in the etiology of stomach cancer in this high risk group.
\end{abstract}

Key Words: Gastric cancer, adenocarcinoma malignant ulcer, early gastric cancer, etiology of gastric cancer, nitrates, upper gestronitestinal tract

\section{Introduction}

The incidence of gastric cancer continues to decline in the United States and throughout much of the World. However, this is not so in the valley of Kashmir located in north-west India. The valley is a stretch of land at an average altitude of 5 to 6 thousand feet above sea level and measures roughly 200 miles $\times 200$ miles. It is bordered on the north-east by the lofty mountains of the Himalayan range and includes the rugged mountain terrain of Ladakh. Its population is million. Muslims constitute $90 \%$ of the population. The incidence of gastric cancer in this group is strikingly high when compared to the prevalence rate in other population groups in other parts of India. This preliminary report describes our experience with gastric cancer at the Sir Maharaja Hari Singh Hospital of the Government Medical College, Srinagar, Kashmir. Our team is also engaged in

From the Division of Gastroenterology, Department of Medicine, Government Medical College, Srinagar, Kashmir, India.

Reprint Requests: Hamid A. Durrani, T-6 Tulshibagh, Srinagar, Kashmir 190009, India. search of 'early gastric cancer'. We will attempt to identify the possible etiological factors in this high risk group. The treatment modalities and results of surgical followup will be documented in a subsequent paper.

\section{Material and methods}

This study comprises 850 patients with gastric cancer diagnosed in the past five years at one teaching-cum-referral hospital. Patients whose clinical presentation warranted a diagnosis or exclusion of gastric cancer by investigations were selected for the study. Symptoms, such as anorexia, weight loss of recent onset, persistent ulcer pain, postprandial abdominal distension, dysphagia, and/or history of upper gastrointestinal (GI) bleed, were considered suggestive of a possible gastric neoplasm. Clinical examination included recording of obvious cachexia, degree of anemia, presence of cervical lymphadenopathy, and of abdominal signs, such as a mass in abdomen, signs of gastric outlet obstruction, hepatomegaly or ascites.

The investigations included routine hemogram, stool examination for occult blood, radiology of upper GI tract including double contrast barium study 
Table 1. Symptomatology

\begin{tabular}{lc}
\hline Symptoms & Percent \\
\hline Dyspepsia-anorexia & 80 \\
Abdominal pain & 12 \\
Upper GI Bleed & 24 \\
Weight loss & 67 \\
Dysphagia & 25 \\
Stasis-obstruction & 8 \\
\hline
\end{tabular}

Table 2. Physical signs

\begin{tabular}{lc}
\hline Physical signs & Percent \\
\hline Emaciation & 52 \\
Anemia & 78 \\
Edema of feet & 12 \\
Clubbing of fingers & 4 \\
Palpable mass & 13 \\
Ascites & 27 \\
\hline
\end{tabular}

of stomach, and upper GI fiberoptic endoscopy with endoscopic biopsy in all patients. The instrument used for the latter procedure was Olympus GIF-D3 or GIF P3. + In a proportion of the patients lactic dehydrogenase ( $\mathrm{LDH}$ ) enzyme activity was measured in serum and gastric juice.

\section{Results}

The majority of patients belonged to the age group 41-60 years. Nine patients were under the age of 20 years, the youngest being a 12 year old boy. All patients below the age of 20 years were males. In the entire series $82 \%$ were males and $18 \%$ females, $62 \%$ were smokers and $38 \%$ non-smokers, $90 \%$ of the smokers were males.

An analysis of the symptomatology showed protean manifestations. However, $80 \%$ of the patients presented with the syndrome of anorexia-dyspepsia of a few weeks to few months duration. Weight loss constituted a major symptom as well (Table 1).

Profound anemia was a major clinical sign in $78 \%$ of patients. All cases were of the hypochromic microcytic type, megaloblastic anemia was seen in none. A palpable mass was present in $13 \%$ of the patients. Ascites was seen in $27 \%$ of the patients, at the time of presentation (Table 2).

The diagnosis of gastric cancer on radiological examination was made on the finding of, 1) a filling defect with a mass lesion, 2) an ulcer with radiological features of malignancy; presence of meniscus with irregular blunting of mucosal folds

†Olympus Optical Company, Tokyo, Japan.
Table 3. Gross Presentation (Endoscopy)

\begin{tabular}{lc}
\hline Morphology & Percent \\
\hline Nodular mass & 47 \\
Polypoid mass & 20 \\
Malignant Ulcer & 10 \\
Infiltrative lesion & 8 \\
Round tumor mass & 8 \\
Linitis plastica & 5 \\
Early gastric cancer & 2
\end{tabular}

Table 4. Histological Typing

\begin{tabular}{lc}
\hline Histological Type & Percent \\
\hline Adenocarcinoma & 79 \\
Mucoid carcinoma & 8 \\
Leiomyosarcoma & 8 \\
Reticulum cell sarcoma & 1 \\
Schirrous carcinoma & 4 \\
\hline
\end{tabular}

around it, and absence of such classic signs of benignity such as Hampton's line, ulcer collar, mound or halo, 3) rigidity, mucosal destruction and decreased distensibility of a segment of the stomach indicating an infiltrative process.

On fiberoptic endoscopy, the commonest site of cancer was the body of stomach $(40 \%)$, followed by the antrum and high lesser curve ( $25 \%$ each). Fundal neoplasm was seen in $5 \%$. Morphological characterestics of cancer were classified on endoscopic examination. A nodular mass was the commonest type of lesion seen in $47 \%$ and a malignant ulcer in $10 \%$. 'Early gastric cancer' was diagnosed in $2 \%$ (Table 3).

On histopathological typing of the specimen obtained fom endoscopic biopsy or on surgery, the commonest type of cancer was found to be adenocarcinoma. Poorly differentiated or well differentiated adenocarcinoma was present in $79 \%$ of the patients (Table 4).

The serum and gastric juice LDH levels were measured in fifty patients with gastric cancer and these were compared with results in patients with benign gastric ulcer and in normal healthy controls. All patients with gastric cancer showed raised levels of the enzyme in both the serum and the gastric juice. (Figure 1), However in patients with schirrois carreinoma, the levels were normal or low.

\section{Discussion}

Gastric cancer is the commonest malignancy involving the GI tract in the valley of Kashmir. Neoplasms of the esophagus are common, but col- 


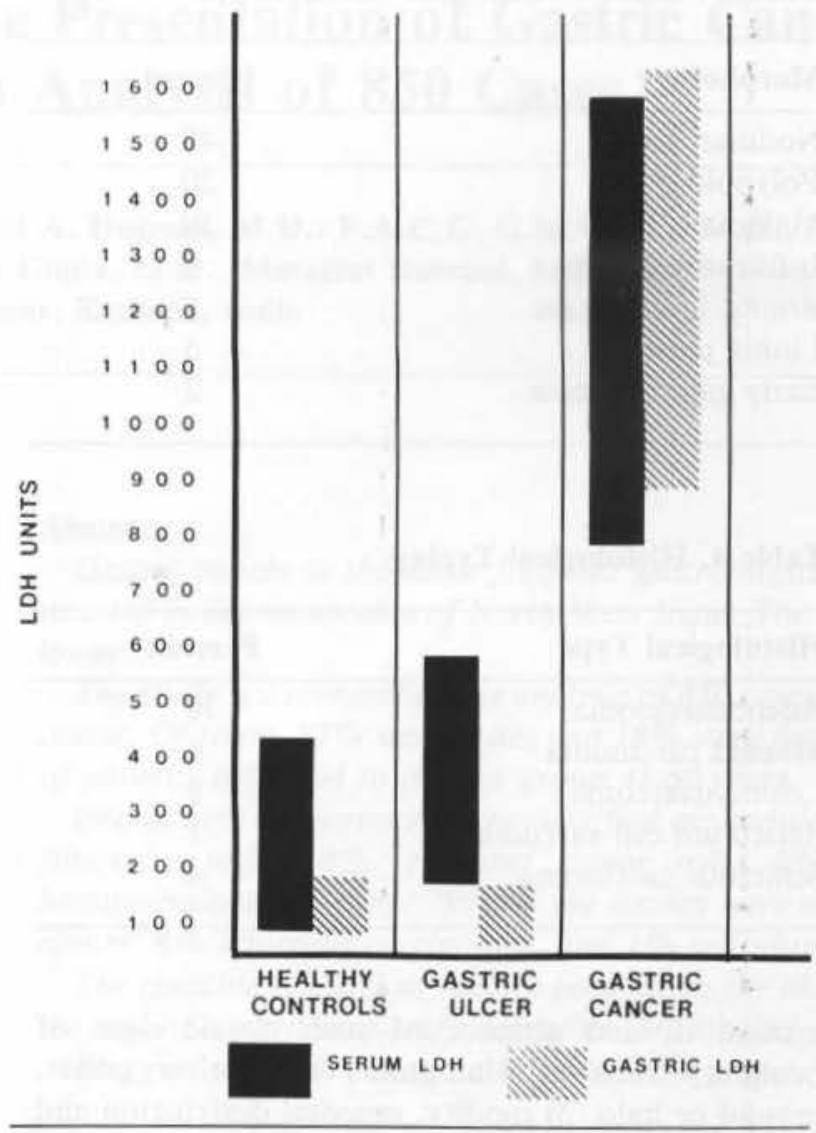

Figure 1

Table 5. Gastrointestinal Neoplasms

Relative Prevalence at SMHS Hospital, Srinagar over the five year period 1982-86

\begin{tabular}{lcc}
\hline Neoplasm & No. of Cases & Percent \\
\hline Esophagus & 460 & 23 \\
Stomach & 850 & 42.7 \\
Jejunum & 24 & 1.2 \\
Colon & 146 & 7.3 \\
Rectum & 190 & 9.5 \\
Biliary & 90 & 4.5 \\
Liver Primary & 44 & 2.5 \\
Secondary & 196 & 9.8 \\
\hline Total & & \\
\hline
\end{tabular}

onic neoplasms are less frequent (Table 5). The present study was conducted in a 500 bed teaching-cumreferral hospital. During the past five years, 850 cases were diagnosed as having gastric cancer, an average of 170 cases registered every year. Gastric cancer formed $3.4 \%$ of all hospital admissions to the medical and the surgical wards. The male to female ratio was $4: 1$. The age incidence was comparable to other sutdies; $88.5 \%$ of the patients were 40 years old

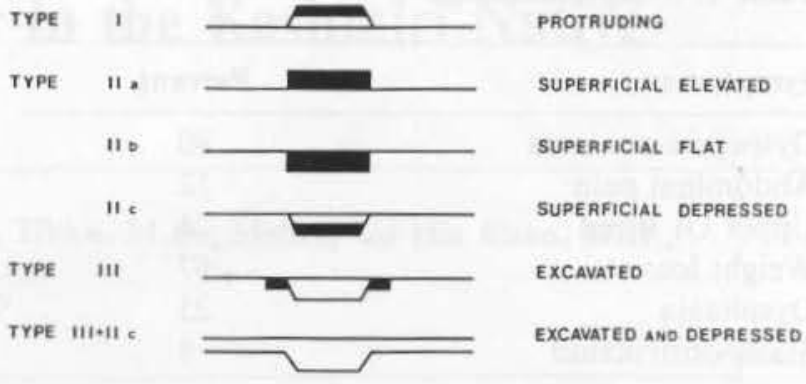

Figure 2

or more. However, a sizable number of 85 patients were in the age group of 20-40 years, and 5 patients, all males, were in their teens. Dyspepsia, anorexia and weight loss constituted the major triad of symptoms. Epigastric pain was not seen as a common symptom and when present it was related to a neoplastic ulcer. About one quarter of the patients presented either with a upper GI bleed or gave a history of recent bleed. Severe anemia, emaciation and evidence of general malnutrition were three important findings on physical examination, and such clinical findings at the time of first reporting speaks of the delay in presentation which added to the problems of surgical resection after diagnosis. When malignant ascites was present in addition, as it was in over a quarter of patients, surgical intevention could not be considered at all. The body of stomach or the middle third was the commonest site, and the nodular mass was the most frequently seen lesion. This is at variance with other works from the Western countries wherein gastric cancer is more often seen in the distal one third of stomach. The large majority of cancers $(87 \%)$ were either simple adenocarcinomas or mucoid adenocarcinomas.

'Early gastric cancer' as classified by the Japan Gastro-enterological Endoscopy Society (Figure 2), was picked up in 17 patients. 'Early gastric cancer' is limited to mucosa or the submucosa, and on endoscopy may appear as a local protrusion, shallow erosion or an excavating lesion ${ }^{1-4}$. However, the unfortunate handicap in diagnosing 'early cancer' is the late reporting of patients in a population group the bulk of which is ignorant of even general health problems.

Lactic dehydrogenase is an oxidative enzyme normally present in serum and gastric juice, as well in other organs such as heart, kidney and lymphoid tissue. The normal values are $100-350$ units $/ \mathrm{ml}$ in serum and $50-250$ units $/ \mathrm{ml}$ in gastric juice. Values of $350-500$ units $/ \mathrm{ml}$ were considered borderline and those above 500 units $/ \mathrm{ml}$ definitely elevated, in the absence of cardiac, renal or lymphoid disease. When compared to gastric ulcer patients and normal healthy controls, the LDH levels in both serum and gastric juice were found to be constantly and 
significantly elevated in patients with gastric cancer (Figure 1) However, in schirrous carcinoma the levels were normal or low, possibly a reflection of the scant cellulrity of the tumor.

It is not the purpose of this study to findout the etiological factors, but it is reasonable to single out such factors which are peculiar to the population group. Some of these factors may be operating collectively in the genesis of stomach cancer in the population under study. Gastric cancer was almost exclusively seen in the natives of Kashmir, irrespective of their religion (Muslims and Hindus being in the ratio of 9:1). Historically, both sects had common ancestors. Cancer of the stomach was far less frequently diagnosed in the vast immigrant population of the valley. A few instances of familial incidence were also noted. Thus a strong case is made for the genetic factor. The environment, with a basic altitude of 5 to 6 thousand feet above sea level and with lofty mountains all around and with severe snow-clad winter for a least four months a year, is not very different from the environment of the North American country of Canada. Of course the luxury of central heating is not available to the vast majority of people. Another environmental factor is the peculiarity of dietary habits in the group under study. The Kashmiri native is exclusively a rice eater, the quantity of rice consumed by an average native is, by all standards, a very large amount which may lead to delayed emptying of the stomach contents. In fact, the stomach as seen on radiology in this population group is a long stomach not uncommonly seen hanging low down at the pelvic brim. The other food item commonly consumed with rice is a green leafy vegetable with broad leaves, called "hak". In addition, the affluent over indulge in lamb meat consumption with a high blend of red chillies. Yet another dietary peculiarity is the "salt tea" beverage, which is a decoction of tea leaves with liberal blend of salt, and is gulped cups after cups at boiling temperatures, and serves as a direct irritant to the mucosa of lower esophagus and the upper stomach.

At present we are not in a position to answer which of these factors could be linked to the genesis of gastric cancer in this high risk population group. It will be interesting to estimate the nitrate content of the most commonly consumed green vegetable and of the water and soil in this population group. Consumption of nitrates has been incriminated as a possi- ble etiological factor in the genesis of gastric cancer given favorable conditions, viz, atrophic gastritis or n-nitrosation deficiency ${ }^{3,6}$. In Japan and Columbia, two countries with the highest prevalence rates of gastric cancer, Japanese soya sauce, preserved fish and fava beans have been shown to act as direct mutagens $^{7-10}$.

Cost factors have hampered further study in defining the etiology, as well as in diagnosing early gastric cancer. Mass endoscopic surveys of healthy population over the age of forty is the primary hope for detecting early gastric cancer and its treatment. This may result in a high five-year survival rate or even cure, following surgery, of this lethal malady.

\section{References}

1. Goldsmith H S, Ghosh B C: Carcinoma of the stomach Am J Surg 1970; 120: 317.

2. Mitsuru K et al: Roentgen features of the superficial depressed type of early gastric cancer. Radiology 1975; 115:289-292

3. Kidokori T, et al: Progress of gastric carcinoma, diagnosis and long term surgical results of early carcinoma. Acta Endocopica, 1981, Toma XI-N2.

4. Japan Research Society for Gastric Cancer; General rules for the gastric cancer study in surgery and pathology. Jap J Surg 1981; 2:2

5. Forman, D: Gastric cancer, diet, and nitrate exposure. Br Med J 1987; 294:528

6. Mirvish SS, Wallcave L, Eagen M, et al: Asorbate-nitrite reactions: a possible means of blocking the formation of carcinogenic N-nitroso compounds. Science 1972; 177:65-8

7. Wakabayshi $\mathrm{K}$, Ochiai $\mathrm{M}$, Saito $\mathrm{H}$, et al, Presence of 1-methyl-1,2,3,4-tetrahydro-betacaroline-3-carboxylic acid, a precursor of a mutagenic nitroso compound, in soy sauce. Proc Natl Acad, Sci, USA 1983;80:2912-16

8. Weisurger $\mathrm{JH}$, Horn CL: Human and laboratory studies on the causes of gastro-intestinal cancer. Scand J Gastroenterol 1984;104:15-26

9. Yang D, Tannenbaum SR, Buchi G,et al: 4-chloro-6 methoxinodole is the precursor of a potent mutagen that forms during nitrosation of the fave bean. Carcinogenesis 1984;5:1219-24

10. Correa P, Cuello C, Fajardo LF, et al: Diet and gastric cancer. Nutrition survey in a high risk area. JNCI 1983;70:673-8. 